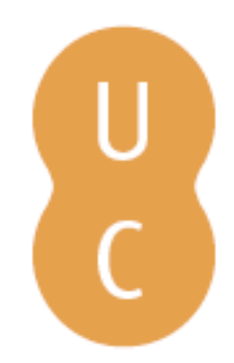

\title{
pommalina
}

\section{Aplicação de tecnologia mineira na separação de plásticos para reciclagem}

Autor(es): $\quad$ Rocha, P. C.; Andrade, L. F.

Publicado por: Imprensa da Universidade de Coimbra

URL

persistente: $\quad$ URI:http://hdl.handle.net/10316.2/31477

DOI: $\quad$ DOI:http://dx.doi.org/10.14195/978-989-26-0531-9_36

Accessed : $\quad$ 26-Apr-2023 04:09:23

A navegação consulta e descarregamento dos títulos inseridos nas Bibliotecas Digitais UC Digitalis, UC Pombalina e UC Impactum, pressupõem a aceitação plena e sem reservas dos Termos e Condições de Uso destas Bibliotecas Digitais, disponíveis em https://digitalis.uc.pt/pt-pt/termos.

Conforme exposto nos referidos Termos e Condições de Uso, o descarregamento de títulos de acesso restrito requer uma licença válida de autorização devendo o utilizador aceder ao(s) documento(s) a partir de um endereço de IP da instituição detentora da supramencionada licença.

Ao utilizador é apenas permitido o descarregamento para uso pessoal, pelo que o emprego do(s) título(s) descarregado(s) para outro fim, designadamente comercial, carece de autorização do respetivo autor ou editor da obra.

Na medida em que todas as obras da UC Digitalis se encontram protegidas pelo Código do Direito de Autor e Direitos Conexos e demais legislação aplicável, toda a cópia, parcial ou total, deste documento, nos casos em que é legalmente admitida, deverá conter ou fazer-se acompanhar por este aviso. 



\section{APLICAÇÃO DE TECNOLOGIA MINEIRA NA SEPARAÇÃO DE PLÁSTICOS PARA RECICLAGEM}

\section{MINERAL PROCESSING TECHNIQUES IN THE SEPARATION OF PLASTICS FOR RECYCLING}

Resumo - A generalidade dos processos de beneficiação de minérios é antiga, baseia-se nas propriedades dos minerais a separar e têm permitido beneficiar todo o tipo de minérios. Os plásticos constituem o terceiro constituinte mais importante dos resíduos sólidos urbanos (RSU), contribuindo com cerca de 10\% do seu peso e cerca de $20 \%$ do seu volume. Embora os plásticos sejam largamente utilizados, consequência das suas excelentes propriedades, eles são maus de reciclar porque são difíceis de separar entre si.

Neste trabalho compara-se a separação por jigagem e por flutuação por espumas de três misturas de plásticos (PS/PMMA, PS/PET e PS/PVC), contribuindo cada um com 50\%, e analisa-se a influência do calibre e forma das partículas.

Para a mistura PS/PMMA a qualidade da separação melhora com o aumento do calibre das partículas nos dois processos. Para os lotes inferiores a 2,8 $\mathrm{mm}$ a jigagem permitiu obter melhores resultados que a flutuação, mas para as fraçóes grosseiras os dois processos conduziram a separaçôes com qualidade semelhante.

A mistura PS/PET apresenta os piores resultados e ambos os processos conduziram a qualidades semelhantes, não sendo evidente a influência do calibre, como consequência do efeito da forma lamelar das partículas de PET.

Para a mistura PS/PVC a flutuaçáo por espumas apresenta melhores resultados do que a jigagem. A qualidade de separação melhora com o aumento do calibre das partículas.

A jigagem e a flutuação por espumas têm potencialidades para poderem ser utilizados como métodos de separação de plásticos.

Palavras-chave: Plásticos; jigagem; flutuação por espumas; calibre das partículas.

\footnotetext{
${ }^{1}$ Departamento de Ciências da Terra da FCTU, Centro de Geociências; fpita@dct.pt
} 
Abstract - The generality of the mineral processing techniques are ancient and they are based on different properties of minerals to separate. Plastics are the third most important component of municipal solid waste (MSW), contributing about $10 \%$ of its weight and about 20\% of its volume. Although plastics are widely used, because of its excellent properties, they are bad to recycle because they are difficult to separate from each other.

In this work jigging and froth flotation separation of three bi-component plastics mixtures (PS/PMMA, PS/PET and PS/PVC) is compared. The effect of the particle sizes was studied in five grain-size fractions.

The quality of the separation by jigging and by froth flotation varies with the plastic mixture and depends on particle size.

For a PS/PMMA mixture, the quality of the separation by the two processes improves with particle size. Grain-size fractions lower than $2.8 \mathrm{~mm}$ have better results by jigging separation than by froth flotation. In the coarser fractions the two processes led to separations with the same quality.

PS/PET mixture presents the worst results of the three mixtures. The two processes led to similar separations. The influence of particle size in the two separation processes was not noticeable, because of the effects of PET particles shape.

In the PS/PVC mixture the froth flotation presents better results than the jigging process. This mixture presented also the best results. The jigging separation improves with particle size.

Jigging and froth flotation have proved to be reliable methods for the separation of plastics.

Keywords: Plastics; jigging; froth flotation; particle size.

\section{1 - Introduçáo}

Os resíduos sólidos urbanos (RSU) constituem uma preocupação ambiental mundial, pois se não forem corretamente tratados provocam a degradação do ambiente e têm efeitos diretos e indiretos na saúde. Porém, para o cidadão comum o problema dos resíduos acaba a partir do momento em que os colocam no contentor coletivo. Nada mais falso, pois começa ali um conjunto de preocupaçóes e de problemas para as entidades responsáveis pela gestão dos RSU.

A correta gestão dos RSU deve obedecer a uma hierarquia de princípios, desde a redução da produção, a reutilização, a reciclagem, a valorização, a destruição e a sua colocação em aterro. A reciclagem é um excelente método de poupança de recursos quando não for possível assegurar a reutilização dos produtos ou, melhor ainda, evitar a produção do resíduo. Mas há algumas limitaçôes ao seu êxito, nomeadamente a separação eficaz dos diferentes tipos de constituintes dos resíduos.

O crescimento demográfico das últimas décadas, a concentração da população nos grandes centros urbanos, a alteração dos hábitos de consumo e o desenvolvimento económico e industrial, têm conduzido à produção de elevadas quantidades de resíduos. Em Portugal a produção de RSU tem aumentado cerca de 3\% ao ano. Em 2010 a produção foi 5183569 toneladas, correspondendo a uma capitação de 1,4 kg/habitante.dia. Naquele ano, 64,3\% dos resíduos foram colocados em aterro, $18 \%$ sofreram valorização energética, 7,6\% sofreram valorização orgânica e 10,1\% foram reciclados. 
Nas últimas décadas os plásticos adquiriram uma grande importância no dia-a-dia, contribuindo para a melhoria do nível de vida das pessoas. A sua ampla utilizaçáo nos mais diversos fins resulta da sua versatilidade e excelentes propriedades. Em Portugal a contribuição dos plásticos nos RSU é de $10 \%$ em peso, representado cerca de $20 \%$ do seu volume.

Ao longo do tempo os plásticos ganharam uma reputação negativa, dada a dificuldade de se decomporem. Porém, tal como a maioria dos constituintes dos RSU também os plásticos podem ser valorizados através da sua reciclagem. Mas para serem reciclados eles têm de ser separados dos restantes constituintes e também têm de ser separados entre si, o que constitui uma dificuldade.

Com o objetivo de facilitar a separação de misturas de plásticos através da utilização de meios mecânicos, têm vindo a ser desenvolvidos estudos com vista à aplicação de técnicas de separação utilizadas na indústria mineira (SHEN et al., 1999, 2001, 2002; SHIBATA et al,. 1996, FERRARA, 2000; AGANTE et al., 2004; FRAUNHOLCZ, 2004; TSUNEKAWA, 2005; DODBIBA et al., 2005; PASCOE, 1999, 2006; ITO et al., 2010; KANGAL, 2010; GENT et al. 2009, 2011; KUWAYAMA, 2011). Com efeito, muitas dessas técnicas estão perfeitamente desenvolvidas e são testadas em larga escala desde há muito tempo, como os processos de separação hidrogravítica (mesas, meios densos, jigagem, hidrociclones), que se baseiam em diferenças de densidade; o processo de flutuação por espumas, que se baseia na hidrofobicidade das partículas, e os processos elétricos, que se baseiam nas propriedades elétricas dos materiais a separar.

No presente estudo aplicam-se duas destas técnicas (jigagem e flutuação por espumas) à separação de três misturas de plásticos. Simultaneamente faz-se também a análise da influência do calibre e da forma das partículas.

\section{2 - Materiais e Métodos}

Para este estudo utilizaram-se quatro tipos de plástico: PS (Poliestireno), PMMA (Polimetil metacrilato) PET (Polietileno tereftalato) e PVC (Policloreto de vinilo), cujas densidades são representadas na Tabela 1 .

Tabela 1 - Densidade dos plásticos utilizados.

\begin{tabular}{cc}
\hline Tipos de plástico & Densidade $\left(\mathrm{kg} / \mathrm{m}^{3}\right)$ \\
\hline PS & 1047 \\
PMMA & 1204 \\
PET-S & 1372 \\
PVC-M & 1326 \\
\hline
\end{tabular}

De modo a verificar a influência do calibre das partículas no processo de separação, analisou-se o comportamento de cinco fraçóes granulométricas (1-1,4; 1,4-2; 2-2,8; 2,8-4 e 4-5,6 mm), cuja forma foi descrita pelo fator forma $\mathrm{F}=\mathrm{D}_{\min } / \mathrm{D}_{\text {max }}$ (onde $\mathrm{D}_{\text {min }}$ e $\mathrm{D}_{\text {máx }}$ representam o diâmetro mínimo e máximo das partículas).

As partículas de PMMA têm uma forma mais regular, similar a pequenas esferas, enquanto que as partículas de PET apresentam uma forma menos regular, semelhantes 
a pequenas lamelas, o que pode condicionar o seu comportamento nos processos de separação, em particular na jigagem, dificultando a sua penetração no leito de jigagem (Fig. 1).

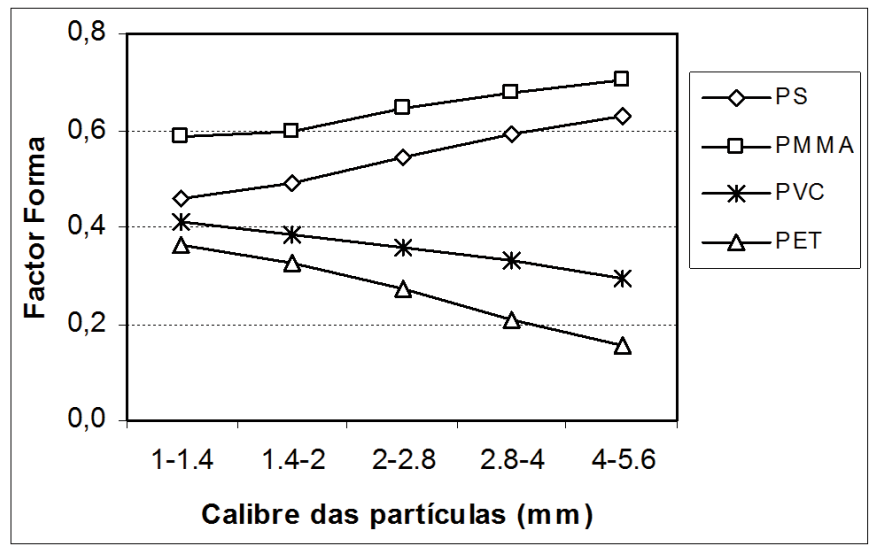

Fig. 1 - Factor forma dos quatro tipos de plásticos e das cinco fracçóes granulométricas.

As separações por jigagem e por flutuação por espumas foram realizadas sobre misturas bicomponente: PS/PMMA, PS/PET, PS/PET e PS/PVC, contribuindo cada plástico com $50 \%$ do peso. As misturas utilizadas tinham proporçóes idênticas de todas as fraçōes granulométricas referidas (entre 1 e $5,6 \mathrm{~mm}$ ).

Os ensaios de jigagem foram realizados numa jiga Denver com secção retangular $(10 \times 15 \times 10 \mathrm{~cm})$. Os ensaios foram conduzidos de modo a obter um afundado o mais expurgado possível de PS, pois este tem menor densidade que os outros três plásticos. O plástico PS saiu por transbordo ("overflow”), constituindo o flutuado, e os outros plásticos ficaram retidos na célula de jigagem. Os ensaios de jigagem foram prologados no tempo de modo a fazer sair por transbordo (flutuado) quase todo o PS.

Os ensaios de flutuação por espumas foram realizados numa célula Denver com uma capacidade de $3 \mathrm{dm}^{3}$. A velocidade de rotaçáo do impulsor foi de $600 \mathrm{rpm}$ e os reagentes químicos utilizados foram o metilisobutilcarbinol (MIBC), como espumante, e o ácido tânico como depressor dos plásticos. A concentração ideal de ácido tânico, que conduziu a separaçóes mais eficientes de cada mistura bicomponente de plásticos, foi selecionada a partir de ensaios previamente realizados. A duração de cada ensaio foi de 8 minutos.

Para comparar a qualidade de separação dos dois processos foi determinada a eficiência de separação definida por SCHULZ (1970) pela expressão $\eta=R_{p 1}-R_{p 2}$, (onde $\eta$ é a eficiência, $\mathrm{R}_{\mathrm{P} 1}$ é a recuperação do plástico 1 no flutuado e $\mathrm{R}_{\mathrm{P} 2}$ é a recuperação do plástico 2 no flutuado).

Nos dois processos o controlo da separação foi realizado a partir da recuperação e do teor de cada tipo de plástico no flutuado e no afundado. Estes parâmetros foram obtidos após separaçáo manual e pesagem dos dois tipos de plásticos presentes no flutuado e afundado, pois eles apresentam cores diferentes. 


\section{1 - Mistura PS/PMMA}

A recuperação e o teor de cada um dos dois plásticos da mistura PS/PMMA no flutuado e afundado dos dois processos de separação e das cinco frações granulométricas são representados na Tabela 2 .

Tabela 2 - Resultados da separação por jigagem e por flutuação da mistura PS/PMMA para cinco fracçôes granulométricas.

\begin{tabular}{|c|c|c|c|c|c|c|c|c|c|}
\hline & \multirow{3}{*}{$\begin{array}{c}\text { Fracçôes } \\
(\mathrm{mm})\end{array}$} & \multicolumn{4}{|c|}{ Recuperação } & \multicolumn{4}{|c|}{ Teor } \\
\hline & & \multicolumn{2}{|c|}{ Flutuado } & \multicolumn{2}{|c|}{ Afundado } & \multicolumn{2}{|c|}{ Flutuado } & \multicolumn{2}{|c|}{ Afundado } \\
\hline \multirow{6}{*}{ 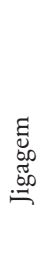 } & & PS & PMMA & PS & PMMA & PS & PMMA & PS & PMMA \\
\hline & $1,0-1,4$ & 99,60 & 32,57 & 0,40 & 67,43 & 75,36 & 26,64 & 0,59 & 99,41 \\
\hline & $1,4-2,0$ & 99,63 & 27,64 & 0,37 & 72,36 & 78,28 & 17,72 & 0,51 & 99,49 \\
\hline & $2,0-2,8$ & 99,62 & 17,78 & 0,38 & 88,22 & 89,43 & 11,57 & 0,42 & 99,58 \\
\hline & $2,8-4,0$ & 99,66 & 7,34 & 0,34 & 92,66 & 93,14 & 6,86 & 0,37 & 99,63 \\
\hline & $4,0-5,6$ & 99,66 & 5,48 & 0,34 & 94,58 & 94,84 & 5,16 & 0,36 & 99,64 \\
\hline \multirow{5}{*}{ 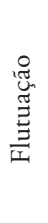 } & $1,0-1,4$ & 97,34 & 88,42 & 2,66 & 11,58 & 52,40 & 47,60 & 18,69 & 81,31 \\
\hline & $1,4-2,0$ & 96,85 & 66,43 & 3,15 & 33,57 & 59,32 & 40,68 & 8,59 & 91,41 \\
\hline & $2,0-2,8$ & 95,75 & 29,60 & 4,25 & 70,40 & 76,38 & 23,62 & 5,69 & 94,31 \\
\hline & $2,8-4,0$ & 94,76 & 5,02 & 5,24 & 94,98 & 94,97 & 5,03 & 5,23 & 94,77 \\
\hline & $4,0-5,6$ & 93,41 & 0,00 & 6,59 & 100,00 & 100,0 & 0,00 & 6,18 & 93,82 \\
\hline
\end{tabular}

Para as três fraçóes finas, a jigagem apresenta melhores resultados do que a flutuação, ela conduziu a maiores recuperaçóes de PS no flutuado e também maiores recuperaçóes de PMMA no afundado, originando um flutuado mais enriquecido em PS e um afundado mais enriquecido em PMMA. Para a fracção fina (1-1,4 mm) a jigagem permitiu obter um flutuado com um teor de 75,36\% em PS, enquanto que na flutuação o flutuado tem um teor de apenas $52,4 \%$ em PS, semelhante ao da mistura inicial (50\%). Relativamente ao afundado, para aquela fração a jigagem permitiu obter um concentrado de PMMA com um teor de 99,4\%, enquanto que na flutuação o afundado apresenta um teor de 81,3\% em PMMA.

Para as duas fraçôes grosseiras os dois processos conduziram a separaçóes com semelhante qualidade. Para a fracção 4-5,6 $\mathrm{mm}$ na jigagem obteve-se um flutuado com 94,8\% em PS e um afundado com um teor de 99,6\% em PMMA enquanto por flutuação se obteve um flutuado puro de PS e um afundado com 93,8\% em PMMA. Assim, para aquela fração, enquanto na jigagem se consegue um afundado quase puro em PMMA, na flutuaçáo consegue-se um flutuado puro em PS.

Nos dois processos de separação, esta melhora com o aumento do calibre das partículas. Para as cinco fraçóes granulométricas, o afundado da jigagem é um produto quase 
puro de PMMA, porque se prolongou os ensaios de jigagem de modo a fazer sair por transbordo (flutuado) quase todo o PS.

\section{2 - Mistura PS/PET}

Nesta mistura, os dois processos de separação conduziram a resultados semelhantes. Verifica-se que o calibre das partículas não apresenta uma significativa influência na qualidade da separação (Tabela 3).

Tabela 3 - Resultados da separação por jigagem e por flutuação da mistura PS/PET para cinco fracçôes granulométricas.

\begin{tabular}{|c|c|c|c|c|c|c|c|c|c|}
\hline & \multirow{3}{*}{$\begin{array}{l}\text { Fracçôes } \\
(\mathrm{mm})\end{array}$} & \multicolumn{4}{|c|}{ Recuperação } & \multicolumn{4}{|c|}{ Teor } \\
\hline & & \multicolumn{2}{|c|}{ Flutuado } & \multicolumn{2}{|c|}{ Afundado } & \multicolumn{2}{|c|}{ Flutuado } & \multicolumn{2}{|c|}{ Afundado } \\
\hline \multirow{6}{*}{ 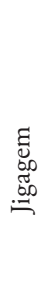 } & & PS & PET & PS & PET & PS & PET & PS & PET \\
\hline & $1,0-1,4$ & 99,69 & 31,02 & 0,31 & 69,98 & 76,86 & 23,14 & 0,44 & 99,56 \\
\hline & $1,4-2,0$ & 99,45 & 26,28 & 0,55 & 73,72 & 79,10 & 20,90 & 0,74 & 99,26 \\
\hline & $2,0-2,8$ & 99,47 & 26,11 & 0,53 & 73,89 & 79,21 & 20,79 & 0,71 & 99,29 \\
\hline & $2,8-4,0$ & 99,64 & 25,77 & 0,36 & 74,23 & 79,45 & 20,55 & 0,48 & 99,52 \\
\hline & $4,0-5,6$ & 99,57 & 28,30 & 0,43 & 71,70 & 77,87 & 22,13 & 0,60 & 99,40 \\
\hline \multirow{5}{*}{ 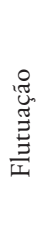 } & $1,0-1,4$ & 96,34 & 25,62 & 3,66 & 74,38 & 78,99 & 21,01 & 4,69 & 95,31 \\
\hline & $1,4-2,0$ & 96,48 & 24,01 & 3,52 & 75,99 & 80,08 & 19,92 & 4,43 & 95,57 \\
\hline & $2,0-2,8$ & 95,48 & 23,34 & 4,52 & 76,66 & 80,36 & 19,64 & 5,57 & 94,43 \\
\hline & $2,8-4,0$ & 94,87 & 21,05 & 5,13 & 78,95 & 81,84 & 18,16 & 6,10 & 93,90 \\
\hline & $4,0-5,6$ & 93,43 & 22,23 & 6,57 & 77,77 & 80,78 & 19,22 & 7,79 & 92,21 \\
\hline
\end{tabular}

Nos dois processos de separação obtêm-se flutuados com aproximadamente $80 \%$ de PS. Para as cinco fraçóes a jigagem permitiu obter afundados quase puros em PET e flutuados com cerca de $78 \%$ de PS. No processo de flutuação obtiveram-se flutuados com um teor em PS ligeiramente superior, mas afundados com teor em PET ligeiramente inferior (aproximadamente 95\%).

\section{3 - Mistura PS/PVC}

$\mathrm{Na}$ Tabela 4 apresentam-se as recuperaçôes e teores do flutuado e afundado da separação por jigagem e por flutuação por espumas para as cinco fraçóes granulométricas da mistura PS/PVC. Em ambos os processos a separação melhora ligeiramente com o aumento do calibre das partículas, sendo este comportamento mais significativo para a jigagem. A recuperação de PVC no flutuado diminui com o aumento do calibre das partículas. 
Tabela 4 - Resultados da separação por jigagem e por flutuação da mistura PS/PVC para cinco fracçōes granulométricas.

\begin{tabular}{|c|c|c|c|c|c|c|c|c|c|}
\hline & \multirow{3}{*}{$\begin{array}{c}\text { Fracçôes } \\
(\mathrm{mm})\end{array}$} & \multicolumn{4}{|c|}{ Recuperação } & \multicolumn{4}{|c|}{ Teor } \\
\hline & & \multicolumn{2}{|c|}{ Flutuado } & \multicolumn{2}{|c|}{ Afundado } & \multicolumn{2}{|c|}{ Flutuado } & \multicolumn{2}{|c|}{ Afundado } \\
\hline \multirow{6}{*}{ 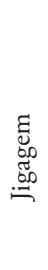 } & & PS & PVC & PS & PVC & PS & PVC & PS & PVC \\
\hline & $1,0-1,4$ & 99,71 & 21,23 & 0,29 & 78,77 & 82,45 & 17,55 & 0,37 & 99,63 \\
\hline & $1,4-2,0$ & 99,55 & 16,75 & 0,45 & 83,25 & 85,60 & 14,40 & 0,54 & 99,46 \\
\hline & $2,0-2,8$ & 99,49 & 13,09 & 0,51 & 86,91 & 88,37 & 11,63 & 0,58 & 99,42 \\
\hline & $2,8-4,0$ & 99,48 & 11,51 & 0,52 & 88,49 & 89,63 & 10,37 & 0,58 & 99,42 \\
\hline & $4,0-5,6$ & 99,38 & 9,61 & 0,62 & 90,39 & 91,18 & 8,82 & 0,68 & 99,32 \\
\hline \multirow{5}{*}{ 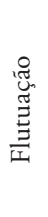 } & $1,0-1,4$ & 97,69 & 6,75 & 2,31 & 93,25 & 93,54 & 6,46 & 2,41 & 97,59 \\
\hline & $1,4-2,0$ & 98,04 & 3,45 & 1,96 & 96,55 & 96,60 & 3,40 & 1,99 & 98,01 \\
\hline & $2,0-2,8$ & 96,90 & 1,69 & 3,10 & 98,31 & 98,29 & 1,71 & 3,06 & 96,94 \\
\hline & $2,8-4,0$ & 96,25 & 1,20 & 3,75 & 98,80 & 98,77 & 1,23 & 3,66 & 96,34 \\
\hline & $4,0-5,6$ & 93,59 & 0,55 & 6,41 & 99,45 & 99,42 & 0,58 & 6,05 & 93,95 \\
\hline
\end{tabular}

Para as cinco fraçóes granulométricas, a flutuação permitiu obter separaçóes ligeira-mente superiores, fundamentalmente nas fraçóes finas. Para a fração fina na flutuação obteve-se um flutuado com um teor de $93,5 \%$ em PS e um afundado com um teor de 97,6\% em PVC, enquanto que na jigagem se obteve um flutuado com um teor de $82,4 \%$ em PS e um afundado com um teor de 99,6\% em PVC.

Para a fração grosseira (4-5,6 mm) obteve-se um flutuado quase puro de PS (99,4\%) na flutuação e um afundado com um teor de $94 \%$ em PVC e na jigagem obteve-se um flutuado com um teor de $91,2 \%$ em PS e um afundado com um teor de 99,3\% em PVC.

\section{4 - Eficiência de separação}

Nos dois processos, a mistura PS/PET é a que apresenta a menor eficiência de separação (Fig. 2). A eficiência da separação de mistura PS/PMMA melhora com o aumento do calibre das partículas, em particular na flutuação, como consequência do aumento da massa das partículas, que torna menor o seu arrastamento. Na célula de flutuação a polpa (mistura de plásticos + água) é sujeita a agitação e as partículas finas de PMMA são mais facilmente arrastadas e recolhidas no flutuado, penalizando a qualidade da separação. A jigagem permite obter melhores resultados para as três fracçóes finas.

$\mathrm{Na}$ mistura PS/PET o calibre das partículas tem uma influência mínima na qualidade da separação. Para as três fraçóes intermédias obtém-se uma eficiência de separação de aproximadamente $73 \%$, valor inferior ao obtido para as outras duas misturas de plásticos. A não melhoria da qualidade de separação com o aumento do calibre das partículas, é consequência da forma lamelar das partículas de PET.

Para a mistura PS/PVC a eficiência de separaçáo, em particular na jigagem, aumenta com o calibre das partículas. A flutuação permite obter uma eficiência de separação ligeiramente superior à da jigagem (aproximadamente 95\%). 


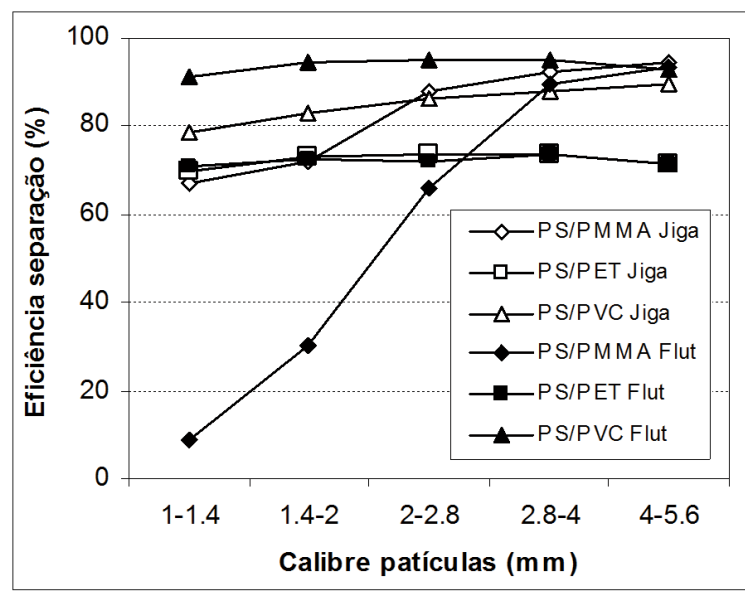

Fig. 2 - Influência do calibre das partículas na eficiência de separação.

Uma vez que a jigagem é um processo hidrogravítico, que se baseia na diferença de densidade dos materiais a separar, esperavam-se os melhores resultados para a mistura PS/ PET e os piores resultados para a mistura PS/PMMA, pois o PET tem a maior densidade e o PMMA tem a menor densidade (Tabela 1). Porém, a jigagem da mistura PS/PET apresenta os piores resultados. Assim, este comportamento não pode ser explicado pela diferença de densidades, devendo ser consequência da forma lamelar das partículas de PET. Este tipo de partículas tem maior dificuldade em penetrar o leito de jigagem, saindo mais facilmente por transbordo, e gerando flutuados menos puros em PS. Este comportamento é mais evidente para as frações grosseiras, sendo os flutuados menos puros em PS.

\section{4 - Conclusóes}

A qualidade da separação operada na jigagem e na flutuação por espumas depende do tipo de mistura e do calibre e forma das partículas.

Para partículas de forma mais regular (isométrica) a qualidade da separação melhora com o aumento do calibre das partículas. Para partículas lamelares essa relação é menos evidente.

Na mistura PS/PMMA, a jigagem permitiu obter melhores resultados, em particular para as fraçóes finas. Para as duas fraçôes grosseiras os dois processos conduziram a resultados semelhantes. A qualidade de separaçáo, em particular na flutuação, melhorou claramente com o aumento do calibre das partículas. Das três misturas analisadas a separação por flutuação da mistura PS/PMMA, das partículas com calibre inferior a $2 \mathrm{~mm}$, apresenta os piores resultados.

Para os lotes inferiores a $2,8 \mathrm{~mm}$ a jigagem conduziu a melhores resultados.

$\mathrm{Na}$ mistura PS/PET, não se observou a predominância de um dos dois métodos de separação. Também não se observou uma clara influência do calibre das partículas nos dois processos de separação. A qualidade da separação desta mistura é pequena, sendo a eficiência de separação inferior a 75\%. 
A mistura PS/PVC apresenta os melhores resultados. Para as cinco fraçóes granulométricas a flutuação por espumas conduziu a melhores resultados do que a jigagem. A separação por jigagem melhorou ligeiramente com o aumento do calibre das partículas.

A forma das partículas tem um papel importante, em particular na jigagem. Verificou-se que para partículas de forma regular, a separação melhora com o aumento do calibre das partículas e, para partículas de forma lamelar (PET), a influência do calibre das partículas é mínima. Apesar do PET ser o plástico de maior densidade, na jigagem a mistura PS/PET conduziu aos piores resultados. Neste processo as partículas lamelares de PET saem mais facilmente pelo flutuado ("overflow") do que as partículas de forma regular de PMMA (na mistura PS/PMMA), apesar deste plástico ter menor densidade que o PET.

Os resultados obtidos mostram que estas técnicas mineiras de separação têm um bom potencial para poderem ser utilizadas na separação de plásticos, possibilitando assim a sua reciclagem.

Agradecimentos - Este trabalho foi financiado pelo Estado Português através da FCT - Fundação para a Ciência e a Tecnologia no âmbito do projecto PEst-OE/CTE/ UI0073/2011 do Centro de Geociências.

\section{Referências Bibliográficas}

AGANTE, E., CARVAlHO, M. T., DURÃO, F. \& BÁRTOLO, T. (2004) - Separation of PET, PVC and PS using gravity concentration and froth flotation. Proceedings of the Global Symposium on Recycling, Waste Treatment and Clean Technology (REWAS’04), Madrid, Spain, p. 1691-1700.

DODBIBA, G., SADAKI, J., OKAYA, K., SHIBAYAA, A. \& FUJITA, T. (2005) - The use of air tabling and triboelectric separation for separating a mixture of three plastics. Minerals Engineering, 18, p. 1350-1360.

FERRARA, G., BEVILACQUA, P., LORENZI, L. \& ZANIN, M. (2000) - The influence of particle shape on the dynamic dense medium separation of plastics. International Journal of Mineral Processing, 59, p. 225-235.

FRAUNHOLCZ, N., (2004) - Separation of waste plastics by froth flotation, Review, Part I. Minerals Engineering, 17, p. 261-268.

GENT, M. R, MENENDEZ, M., TORANO, J., ISIDRO, D. \& TORNO, S. (2009) - Cylinder cyclone (LARCODEMS) density media separation of plastic wastes. Waste Management, 29, p. 1819-1827.

GENT, M. R, MARIO, M., JAVIER, T. \& SUSANA, T. (2011) - Optimization of the recovery of plastics for recycling by density media separation cyclones. Resources, Conservation and Recycling, 55, p. 472-482.

ITO, M., TSUNEDKAWA, M., ISHIDA, E., KAWAI, K., TAKAHASHI, T., ABE, N. \& HIROYOSHI, N. (2010) - Reverse jig separation of shredded floating plastics - separation of polypropylene and high density polyethylene. International Journal of Mineral Processing, 97, p. 96-99.

KANGAL, M. O. (2010) - Selective flotation technique for separation of PET and HDPE used in drinking water bottles. Mineral Processing and Extractive Metallurgy Review, 31, p. 214-223.

KUWAYAMA, Y., ITO, M., HIROYOSHI, N. \& TSUNEDKAWA, M. (2011) - Jig separation of crushed automobile shredded residue and its evaluation by float and sink analysis, Journal of Material Cycles and Waste Management, p. 1-7.

PASCOE, R. D. e HOU, Y. Y. (1999) - Investigation of the importance of particle shape and surface wettability on the separation of plastics in a LARCOMEDS separator, Minerals Engineering. 12, p. 423-431.

PASCOE, R. D., (2006) - Investigation of hydrocyclones for the separation of shredded fridge plastics. Waste Management, 26, p. 1126-1132. 
SHEN, H., PUGH, R. J. \& FORSSBERG, E. (1999) - A Review of plastics recycling and the flotation of plastics, Resources, Conservation and Recycling, 25, p. 85-109.

SHEN, H., FORSSBERG, E. H. \& PUGH, R. J. (2001) - Selective flotation separation of plastics by particle control. Resources, Conservation and Recycling, 33, p. 37-50.

SHEN, H. FORSSBERG, E. \& PUGH, R. J. (2002) - Selective Flotation Separation of Plastics by Chemical Conditioning With Methyl Cellulose. Resources, Conservation and Recycling, 35, p. 229-241.

SHIBATA, J., MATSUMOTO, S., YAMAMOTO, H. \& LUSAKA, E. (1996) - Flotation separation of plastics using selective depressants. International Journal of Mineral Processing, 48, p. 127-134.

SCHULZ, N. F., (1970) - Separation efficiency, Transactions SME/AIME. 247, p. 81-87.

TSUNEKAWA, T., NAOI, B., OGAWA, S., HORI, K., HIROYOSHI, N., ITO, M. \& HIRAJIMA, T. (2005)

- Jig separation of plastics from scrapped copy machines. International Journal of Mineral Processing, 76, p. 67-74. 